\title{
Atypical Respiratory Distress Suspect of COVID-19 in Eastern Democratic Republic of the Congo prior to the Pandemic. A Case Report
}

\section{Guy-Quesney Mateso}

Department of Internal Medicine, Hôpital Provincial Général de Référence de Bukavu (HPGRB), Bukavu, DRC

Marius BAGUMA ( $\sim$ baguma.akonkwa@ucbukavu.ac.cd)

Université Catholique de Bukavu (UCB) https://orcid.org/0000-0002-4934-4846

\section{Pacifique Mwene-Batu}

Ecole Régionale de Santé Publique (ERSP), Université Catholique de Bukavu (UCB), Bukavu, DRC

\section{Ghislain Maheshe Balemba}

Faculty of Medicine, Université Catholique de Bukavu (UCB), Bukavu, DRC

\section{Fabrice Nzabara}

Department of Internal Medicine, Hôpital Provincial Général de Référence de Bukavu (HPGRB), Bukavu, DRC

\section{Samuel Makali}

Department of Internal Medicine, Hôpital Provincial Général de Référence de Bukavu (HPGRB), Bukavu, DRC

\section{Aline Bedha}

Department of Internal Medicine, Hôpital Provincial Général de Référence de Bukavu (HPGRB), Bukavu, DRC

\section{Bonheur Furaha}

Department of Internal Medicine, Hôpital Provincial Général de Référence de Bukavu (HPGRB), Bukavu, DRC

\section{Jimmy Minani}

Department of Internal Medicine, Hôpital Provincial Général de Référence de Bukavu (HPGRB), Bukavu, DRC

\section{Christian Tshongo Muhindo}

Faculty of Medicine, Université Catholique de Bukavu (UCB), Bukavu, DRC

\section{Espoir Bwenge Malembaka}

Ecole Régionale de Santé Publique (ERSP), Université Catholique de Bukavu (UCB), Bukavu, DRC

\section{Mannix Imani Masimango}

Faculty of Medicine, Université Catholique de Bukavu (UCB), Bukavu, DRC

Tony Akilimali Shindano 
Faculty of Medicine, Université Catholique de Bukavu (UCB), Bukavu, DRC

\section{Justin Cirhuza Cikomola}

Faculty of Medicine, Université Catholique de Bukavu (UCB), Bukavu, DRC

\section{Kanigula Mubagwa}

Faculty of Medicine, Université Catholique de Bukavu (UCB), Bukavu, DRC

\section{Case report}

Keywords: COVID-19, SARS-Cov-2 coronavirus, pandemic, Democratic Republic of the Congo, DRC, Case report

Posted Date: December 3rd, 2020

DOl: https://doi.org/10.21203/rs.3.rs-117083/v1

License: (c) (1) This work is licensed under a Creative Commons Attribution 4.0 International License. Read Full License

Version of Record: A version of this preprint was published at Tropical Diseases, Travel Medicine and Vaccines on April 6th, 2021. See the published version at https://doi.org/10.1186/s40794-021-00134-6. 


\section{Abstract}

Background: Predictions have been made that Africa would be the most vulnerable continent to the novel Coronavirus disease 2019 (COVID-19). Interestingly, the spread of the disease in Africa seems to have been delayed and initially slower than in many parts of the world. Here we report on two cases which make us suspect that COVID-19 might have been present in our region before the official declaration of the disease in December 2019.

Case presentation: These two cases (one 55-year-old man and one 25-year-old woman) of acute respiratory distress secondary to atypical pneumonia were seen in Bukavu, in Eastern Democratic Republic of the Congo (DRC), between September and December 2019. One patient had returned from China and the other had close contacts with travellers from China in the 2 weeks prior to the onset of symptoms. In either case, the aetiology could not be accurately determined. However, the two cases presented a clinical picture (progressive dyspnoea, preceded by dry cough and fever) and laboratory changes (procalcitonin within the normal range, slight inflammation, and lymphopenia) compatible with a viral infection. The chest X-Rays series of the first patient showed lesions (reticulations, ground glass, and nodules $\leq 6 \mathrm{~mm}$ ) similar to those found in COVID-19. In addition, unlike the 25-year-old female patient who had no comorbidity, the 55-year-old male patient who had hypertension as comorbidity, developed a more severe acute respiratory distress which progressed to death.

Conclusion: These cases bring to the attention a number of facts which make us suspect that COVID-19 may have already been present in the region months before the official beginning of the pandemic. This is important, as prior presence of the disease or infections with related virus may account for the delayed and less extensive development of the epidemic in the region.

\section{Background}

The world is currently facing a devastating pandemic of the novel coronavirus disease 2019 (COVID-19), caused by severe acute respiratory syndrome coronavirus 2 (SARS-CoV-2), which was first declared in Wuhan, China, in December 2019 [1]. Clinically, the infection by the virus is asymptomatic in most cases $[2,3]$. In many cases, it may manifest itself by non-typical symptoms such as fever, cough, sore throat [48], known to be present in common cold. In some other cases more evocative symptoms such as anosmia and ageusia may be present $[9,10]$. Most severe cases develop respiratory distress with hypoxia $[5,6]$. Laboratory findings include an inflammatory syndrome, mild lymphopenia and an increased level of cytokines [11]. Pneumonia is present on chest X-Rays (CXR), and more specific aspects such as ground-glass images can be detected on radiography or CT scan [5-8]. Although the first COVID-19 cases were described in China [4-8], the disease has not spread in the country to a level it has since spread in other countries. As of November 15, 2020, over 53.8 million cases of COVID-19 have been confirmed in 191 countries, including more than 1.31 million deaths [12]. Predictions have been made that Africa would be the most vulnerable continent to COVID-19, because of various factors: dense population in many cities, close physical contacts indoors due to poor housing conditions or outdoors due to the fact 
that many people rely on daily income from small business, high prevalence of chronic diseases (e.g., infections by human immunodeficiency virus (HIV), malnutrition, etc.) interfering with the immune defence, absent or poor infrastructures to diagnose or treat patients, and very frequent travels between China and Africa $[13,14]$. Sub-Saharan African countries are shown to have the world's highest estimated vulnerability index to infectious disease outbreaks [15]. The Democratic Republic of the Congo (DRC) is considered to be among the countries at highest risk, especially since travel continued even after the pandemic was declared [13].

Interestingly, except for South Africa, the spread of the disease in the rest of sub-Saharan Africa seems to have been delayed and relatively slower than in many parts of the world. Since the first COVID-19 case was officially identified in Africa (in Egypt) on February 14, 2020 [16, 17], 1,267,664 confirmed cases and 28,469 deaths have been documented as of November 10,2020, about nine months later [18]. These numbers are far below those recorded in other parts of the world. In the DRC, the first case has been reported on March 10, 2020, in Kinshasa (12 million inhabitants), but as of November 01, 2020, only 8,512 confirmed cases have been registered in the same city and 11,395 countrywide [19]. In Bukavu (about 1 million inhabitants, density: 16,600 inhabitants $/ \mathrm{km}^{2}$ ), the capital city of South-Kivu, in Eastern DRC, the first two COVID-19 cases were travellers who arrived on March 18 and 20, but despite a delay in quarantine measures (started on March 30,2020), seven months later the province has registered only 318 confirmed cases of which 46 deaths [20].

The reasons for the low rate of COVID-19 propagation in Africa remain unknown. In this report, we present two patients who were admitted between September and December 2019 for acute respiratory distress in the Intensive Care Unit (ICU) of the "Hôpital Provincial Général de Référence de Bukavu" (HPGRB), in Bukavu. For both patients, a diagnosis of atypical viral pneumonia was made. Although they were not tested for COVID-19 (as the disease was not yet known at that time) the clinical presentation, radiological findings, and the course of the disease are compatible with COVID-19.

Our report is timely in that it coincides with a moment the debate about herd immunity is rife in many countries in Europe and North America which are grappling with a second wave of COVID-19 and societal impacts of containment measures. Understanding the reasons for the potential presence of the SARSCov-2 coronavirus on the African continent way longer before the pandemic unveiled, without resulting in as severe damages as in Europe and the Americas, may be key both to curb the current course of the disease in highly-burdened countries and to sustain the seemingly downward trends in the COVID-19 spread in Africa, while awaiting the vaccine. Our report also comes to further underpin the urgent call for inclusion of Africa in the current planning and prioritization processes for global COVID-19 research [21].

\section{Methods}

Upon learning of coronavirus, we revisited the files of a few patients who had been hospitalized during the last term of 2019 , and enrolled the two patients presented in this report, who had been admitted with fever, cough and hypoxia with signs of atypical pneumonia on chest radiography. 
We reviewed their medical records to collect information on physical examination, and the laboratory (including haematology, biochemistry, and microbiology tests) as well as the CXR findings. Complete blood count was carried out using an automated haematology analyser (Edan H50, Hamburg, Germany). Biochemical tests on plasma or serum were done using semi-automatic analysers (CYANSmart CY009, CYPRESS Diagnostics, Langdorp, Belgium, for creatinine and blood urea nitrogen; Rayto Chemray 120 , Shenzhen, China, for alanine transaminase, aspartate aminotransferase, alkaline phosphatase, albumin and bilirubin; iCHROMA II, Brussels, Belgium, for D-dimer and procalcitonin; EDAN i15 analyser, Hamburg, Germany, for arterial blood gas). Bacteriological tests included blood cultures performed in BACT/ALERT® FA Plus and BACT/ALERT ${ }^{\circledR}$ FN culture media (bioMerieux, Marcy-l'Etoile, France), screening of tuberculosis using a Ziehl-Neelsen stain of sputum, and microscopic examination of Giemsa-stained thick blood film for diagnosis of clinical malaria. At the time of the hospitalization of the two patients, there was no capacity for regular testing for the presence of respiratory virus. HIV test was carried out using rapid point-of-care test DETERMINE ${ }^{\mathrm{TM}}$ HIV-1/2 AG/AB COMBO (Florida, USA). CXR were carried out using Perlong PL 50DR Digital Radiography (Nanjing, China).

No ethical permission was needed since the study involved a retrospective review of data from already treated patients, whose identity was kept confidential.

\section{Case Presentation}

\section{Case 1}

A 55-year-old man, with a 10-year history of hypertension (treated with nicardipin $20 \mathrm{mg}$ twice daily, bisoprolol $5 \mathrm{mg}$ twice daily and aldactazine 1 tablet once daily) and a 5-year history of pigeon breeding, was seen in the Department of Internal Medicine on September 20, 2019, for dry cough, 2 weeks after returning from Guangzhou (Guangdong province, China). The patient did not complain of any loss of smell or taste. He had good mental orientation. Axillary temperature was $37^{\circ} \mathrm{C}$. Chest auscultation noted normal heart sounds and normal vesicular breath sounds without rales or sibilance. There was no abdominal tenderness nor enlarged liver or spleen. There was no low limb oedema. There was no loss of peripheral motor or sensor function and no other abnormal neurological signs. Laboratory results showed normal complete blood count $(\mathrm{CBC})$ and procalcitonin $(<0.1 \mathrm{ng} / \mathrm{ml})$; an elevated serum creatinine $\left(1.8 \mathrm{mg} / \mathrm{dl}\right.$, hence a glomerular filtration rate, GFR, of $\left.50.6 \mathrm{ml} /\left(\mathrm{min}^{\star} 1.73 \mathrm{~m}^{2}\right)\right)$ and electrolytes disturbances (low sodium at $130 \mathrm{mmol} / \mathrm{l}$, low potassium at $3.4 \mathrm{mmol} / \mathrm{l}$ and low calcium $1.04 \mathrm{mmol} / \mathrm{l}$ ) (Table 1). A diagnosis of non-specific interstitial pneumonia was evoked based on a CXR showing bibasal and posterior densification (25-30\% of total chest height), blurring heart borders and diaphragm, associated with trabecular bands and ground-glass zones on the mid third of the lungs (Fig. 1a, b). He was sent back home with oral azithromycin ( $500 \mathrm{mg}$ daily for five days), paracetamol (1000 $\mathrm{mg}$ up to 3 times a day if necessary) and an antitussive drug. 
Table 1

Summary of laboratory tests for the 55-year-old male patient

\begin{tabular}{|c|c|c|c|c|c|c|c|}
\hline Laboratory tests & $\begin{array}{l}\text { Normal } \\
\text { values }\end{array}$ & $\begin{array}{l}\text { Sept. } \\
20\end{array}$ & Oct. 10 & $\begin{array}{l}\text { Oct. } \\
14\end{array}$ & $\begin{array}{l}\text { Oct. } \\
17\end{array}$ & Oct. 23 & $\begin{array}{l}\text { Oct. } \\
28\end{array}$ \\
\hline \multicolumn{8}{|l|}{ Arterial Blood gas } \\
\hline $\mathrm{pH}$ & $7.35-7.45$ & - & 7.55 & 7.52 & 7.55 & 7.31 & 6.97 \\
\hline $\mathrm{PaO}_{2}(\mathrm{mmHg})$ & $75-100$ & - & 33 & 48 & 49 & 60 & 60 \\
\hline $\mathrm{PCO}_{2}(\mathrm{mmHg})$ & $35-45$ & - & 29.7 & 36.5 & 31.6 & 43 & 74 \\
\hline $\mathrm{SaO}_{2}(\%)$ & $94-100$ & - & 74 & 88 & 89 & 92 & 73 \\
\hline Lactates (mmol/L) & $<2.00$ & - & 1.89 & 4.00 & 3.27 & 3.1 & 12.2 \\
\hline Bicarbonates (mmol/L) & $22.0-26.0$ & - & 25.1 & 28.8 & 27.0 & 21 & 16.5 \\
\hline $\mathrm{P}_{\mathrm{a}} \mathrm{O}_{2} / \mathrm{FiO}_{2}(\mathrm{mmHg})$ & $400-500$ & - & 157 & 229 & 233 & 60 & 60 \\
\hline \multicolumn{8}{|l|}{ Complete blood count } \\
\hline $\begin{array}{l}\text { White blood cells }\left(x 10^{3} /\right. \\
\mu \mathrm{L})\end{array}$ & $4.00-10.00$ & 8.00 & 11.60 & - & - & - & 7.60 \\
\hline Neutrophiles $\left(x 10^{3} / \mu \mathrm{L}\right)$ & $1.50-7.00$ & 5.46 & 9.07 & - & - & - & 5.40 \\
\hline Lymphocytes $\left(x 10^{3} / \mu \mathrm{L}\right)$ & $1.50-4.50$ & 1.63 & 1.38 & - & - & - & 1.43 \\
\hline Monocytes $\left(x 10^{3} / \mu \mathrm{L}\right)$ & $0.20-1.00$ & 0.74 & 1.11 & - & - & - & 0.74 \\
\hline Eosinophiles $\left(x 10^{3} / \mu \mathrm{L}\right)$ & $0.10-0.50$ & 0.16 & 0.02 & - & - & - & 0.03 \\
\hline Red blood cells $\left(x 10^{6} / \mu \mathrm{L}\right)$ & $4.20-5.70$ & 5.28 & 4.90 & - & - & - & 5.41 \\
\hline Hemoglobin $(\mathrm{g} / \mathrm{L})$ & $13.0-18.0$ & 16.0 & 15.3 & 17.0 & - & - & 16.5 \\
\hline Hematocrit (\%) & $40.0-52.0$ & 45.1 & 42.3 & 50.0 & - & - & 48 \\
\hline Platelets $\left(x 10^{3} / \mu \mathrm{L}\right)$ & $150-450$ & 244 & 213 & - & - & - & 156 \\
\hline \multicolumn{8}{|l|}{ Electrolytes } \\
\hline Sodium (mmol/L) & $135-155$ & 130 & 129 & 135 & 132 & - & 133 \\
\hline Potassium (mmol/L) & $3.5-5.1$ & 3.4 & 3.4 & 3.2 & 3.4 & - & 3.7 \\
\hline Chloride (mmol/L) & $96-115$ & 99 & 99 & 102 & 99 & - & 115 \\
\hline Calcium (mmol/L) & $1.10-1.40$ & 1.04 & 1.12 & 1.21 & 1.25 & - & 1.33 \\
\hline Magnesium (mg/dL) & $1.60-2.50$ & - & - & 1.8 & - & - & 1.7 \\
\hline C-Reactive protein (mg/L) & $<3.0$ & - & 54.9 & - & 98 & 43 & 102 \\
\hline
\end{tabular}




\begin{tabular}{|c|c|c|c|c|c|c|c|}
\hline Laboratory tests & $\begin{array}{l}\text { Normal } \\
\text { values }\end{array}$ & $\begin{array}{l}\text { Sept. } \\
20\end{array}$ & Oct. 10 & $\begin{array}{l}\text { Oct. } \\
14\end{array}$ & $\begin{array}{l}\text { Oct. } \\
17\end{array}$ & Oct. 23 & $\begin{array}{l}\text { Oct. } \\
28\end{array}$ \\
\hline Procalcitonin (ng/mL) & $<0.10$ & $<0.10$ & $<0.10$ & - & - & 0.69 & $<$ \\
\hline Creatinine (mg/dL) & $0.6-1.40$ & 1.80 & 2.04 & - & - & 1.80 & 3.8 \\
\hline Blood Urea Nitrogen & $8.0-23.0$ & - & 40.0 & - & - & 107 & 150 \\
\hline Blood Glucose (mg/dl) & $60-110$ & 82 & 127 & - & - & 82 & - \\
\hline Hb1Ac (\%) & $4-5.70$ & 3.29 & - & - & - & - & - \\
\hline Troponin $(\mathrm{ng} / \mathrm{ml})$ & & 0.02 & - & - & - & - & - \\
\hline Pro-BNP (pg/ml) & & - & 183.4 & - & - & 101.5 & - \\
\hline D-dimer ( $\mu \mathrm{g} / \mathrm{L})$ & $<500$ & - & 790 & - & - & - & - \\
\hline Thick blood film & Negative & - & Negative & - & - & Negative & - \\
\hline Hemocultures & Negative & - & Negative & - & - & Negative & - \\
\hline $\begin{array}{l}\text { Ziehl-Neelsen stain of } \\
\text { sputum }\end{array}$ & Negative & - & Negative & - & - & - & - \\
\hline \multicolumn{8}{|l|}{ Serologies } \\
\hline HIV & Negative & - & Negative & - & - & - & - \\
\hline Viral hepatitis B & Negative & - & Negative & - & - & - & - \\
\hline Viral hepatitis C & Negative & - & Negative & - & - & - & - \\
\hline Syphilis & Negative & - & Negative & - & - & - & - \\
\hline
\end{tabular}

Despite treatment, coughing was exacerbated, and fever and dyspnoea occurred two weeks later. On October 10, 2019, he was admitted in the Emergency ward. The clinical evaluation noticed polypnea (30 breaths $/ \mathrm{min})$, tachycardia $(100$ beats $/ \mathrm{min})$ and fever $\left(38^{\circ} \mathrm{C}\right)$. Oxygen saturation $\left(\mathrm{SaO}_{2}\right)$ measured with a pulse oximeter was $54 \%$ [normal range: $95-100 \%$ ]. The patient presented a respiratory distress (nose flaring, chest retractions and cyanosis) and had fine crackles at both lung bases. Cardiovascular examination was normal. Laboratory results showed a moderate inflammatory syndrome with hyperleukocytosis $(11,600$ leucocytes/ $\mu \mathrm{l}[4,000-10,000 / \mu \mathrm{l}]$ of which 9,071 neutrophils $[1,500-7,000 / \mu \mathrm{l}]$

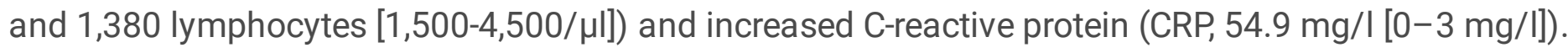
Procalcitonin levels were normal [below $0.1 \mathrm{ng} / \mathrm{ml}$ ]. An arterial blood gas (ABG) analysis revealed the presence of severe hypoxemia $\left(\mathrm{P}_{\mathrm{a}} \mathrm{O}_{2} 33 \mathrm{mmHg}[75-100 \mathrm{mmHg}], \mathrm{SaO}_{2} 74 \%\right)$ and respiratory alkalosis ( $\mathrm{pH}$ 7.55 [7.35-7.45], $\mathrm{P}_{\mathrm{a}} \mathrm{CO}_{2} 29.7 \mathrm{mmHg}$ [35-45 mmHg], $\mathrm{P}_{\mathrm{a}} \mathrm{O}_{2} / \mathrm{FiO}_{2}$ ratio $157 \mathrm{mmHg}$ [400-500 mm Hg], bicarbonate $25.1 \mathrm{mmol} / \mathrm{l}[22-26 \mathrm{mmol} / \mathrm{l}]$, and lactates $1.89 \mathrm{mmol} / \mathrm{l}[<2 \mathrm{mmol} / \mathrm{l}])$. Bacteriological investigations were all negative. Tuberculosis was unlikely given a Ziehl-Neelsen stain of sputum which was negative for three samples collected at different times. HIV serological tests were negative (Table 1). 
A second CXR showed persistence of the aforementioned findings, except for the trabeculae hidden by the alveolar densification that had progressed up to the level of the main bronchi, overhung by a well delimited ground glass zone without encroachment upon the apices (Fig. 1c).

The patient was hospitalized in the ICU for acute respiratory distress syndrome (ARDS) secondary to viral pneumonia with probable bacterial superinfection. He received oxygen by mask ( 5 litres $/ \mathrm{min}$ ) and intravenous antibiotics (combination of amoxicillin and clavulanic acid 1/0.25 g thrice daily). Despite this treatment, continuous fever (average temperature: $38.5^{\circ} \mathrm{C}$ ) and hypoxemia persisted, and lactates increased to $4 \mathrm{mmol} / \mathrm{l}$. So, two days later, amoxicillin and clavulanic acid were replaced by levofloxacin (500 mg) and ceftriaxone (1 g), both twice daily, in addition to intravenous dexamethasone (16 mg thrice daily).

Four days later, no improvement was observed. The oxygen flow was increased to 10 litres $/ \mathrm{min}$ and dexamethasone replaced by methylprednisolone ( $125 \mathrm{mg}$ twice daily for 5 days). During the following week, there was a slight improvement of the dyspnoea and fever, but on October 23, 2019, fever reappeared, and the respiratory distress worsened. A new CXR showed a reduction of the alveolar consolidation to the advantage of an interstitial syndrome (reticulations, ground-glass, and nodules $\leq$ $6 \mathrm{~mm}$ ) extending to lung tops (Fig. 1d). Meanwhile, the CRP was $49 \mathrm{mg} / \mathrm{l}$ and procalcitonin $0.62 \mathrm{ng} / \mathrm{ml}$. A diagnosis of respiratory zoonosis was considered, given the history of pigeon breeding. Intravenous methylprednisolone (125 mg twice daily) was reintroduced and Duovent ${ }^{\circledR}$ (combined ipratropium and fenoterol) was administered in nebulization, without any improvement. $\mathrm{SaO}_{2}$ (measured with a pulse oximeter) remained at $40 \%, \mathrm{P}_{\mathrm{a}} \mathrm{O}_{2} / \mathrm{F}_{\mathrm{i}} \mathrm{O}_{2}$ ratio decreased to less than $70 \mathrm{mmHg}$, and the patient became confused, with loss of notion of space and time, probably because of the hypoxia.

On October 25, 2019, the patient underwent orotracheal intubation for mechanical ventilation. This improved the $\mathrm{SaO}_{2}$ to $90-94 \%$ but on October 28,2019 , the patient developed a shock, with increased lactacidemia $(12.2 \mathrm{mmol} / \mathrm{l})$, followed within a few hours by cardiac arrest irresponsive to resuscitation.

\section{Case 2}

A 25-year-old woman, with no history of recent travel, was admitted at the Emergency ward on December 18,2019 , for a one-week progressive dyspnoea, preceded by dry cough and fever. She had no particular medical history and was a nurse in a hospital where Chinese employees from a multinational mining company are treated, of whom some had recently travelled from China (the place they came from in China could not be determined) and three of them had presented flu-like symptoms within 2 weeks. On admission, she could not complete sentences due to dyspnoea. At ambient air, she presented a $\mathrm{SaO}_{2}$ of $82 \%$ and signs of respiratory distress without cyanosis. Her pulmonary and cardiac auscultation was normal.

The CXR showed reticular lines and peribronchovascular haziness in the infrahilar and retrocardiac regions, bilaterally. This suggested a mild interstitial pneumonia (Fig. 2a, b). Laboratory results showed a slight inflammation with CRP at $14.5 \mathrm{mg} / \mathrm{l}$, lymphopenia (700 lymphocytes/ $\mu \mathrm{l}$ ) and normal procalcitonin 
$(<0.1 \mathrm{ng} / \mathrm{ml})$. The ABG showed a hypoxemia $\left(\mathrm{P}_{\mathrm{a}} \mathrm{O}_{2} 60 \mathrm{mmHg}\right)$ and a respiratory alkalosis $(\mathrm{pH} 7.51$, $\mathrm{P}_{\mathrm{a}} \mathrm{CO}_{2} 35 \mathrm{mmHg}, \mathrm{P}_{\mathrm{a}} \mathrm{O}_{2} / \mathrm{F}_{\mathrm{i}} \mathrm{O}_{2} 286 \mathrm{mmHg}$, bicarbonate $27.1 \mathrm{mmol} / \mathrm{l}$, and lactates $\left.1.50 \mathrm{mmol} / \mathrm{l}\right)$. Creatinine, blood urea nitrogen and blood electrolytes were normal. HIV serology was negative.

A diagnosis of moderate ARDS secondary to a viral pneumonia was retained and the patient was admitted in the ICU, receiving oxygen (4 litres/min), azithromycin (500 mg once daily for 5 days) and Duovent ${ }^{\circledR}$ in nebulization. Three days later, she was eupnoeic with normal $\mathrm{SaO}_{2}$ at ambient air. She was discharged from hospital five days after admission.

\section{Discussion}

In this report, we present two cases of acute respiratory distress secondary to atypical pneumonia in eastern DRC. One patient had returned from China and the other had had close contacts with employees who had returned from a travel to China in the 2 weeks prior to the onset of symptoms. In one case, the respiratory disease did not respond to treatments for typical and atypical causes of respiratory failure. In the second case, hypoxia was disproportionate with the chest lesions. In no case could the aetiology be accurately determined. COVID-19 was not yet known when these patients were hospitalized. However, when viewed retrospectively the two cases presented a clinical picture and laboratory changes (procalcitonin within the normal range, slight inflammation, and lymphopenia) compatible with a viral infection. The CXRs series of the first patient showed lesions similar to those found in COVID-19 [22]. In addition, unlike the 25-year-old female patient who had no comorbidity, the 55-year-old male patient who had hypertension as comorbidity, developed a more severe acute respiratory distress which progressed to death. For COVID-19, available data show that older age and comorbidities such as cardiovascular diseases, diabetes, hypertension, chronic respiratory diseases and cancer are associated with an increased risk of death $[23,24]$. A diagnosis of psittacosis can hardly be sustained in view of the dramatic evolution to death despite the use of antibiotics, including a macrolide and a quinolone, which are known to be efficacious against Chlamydophila psittaci [25]. In retrospect, especially given the context of travel to China or of contact with travellers from China, the question can thus be raised as to whether these cases could have been COVID-19 infections.

The two cases and a number of other facts make us suspect that COVID-19 may have already been present in some regions of eastern DRC before the official beginning of the pandemic. The cases presented here are illustrative of (unpublished) observations made by clinicians in Bukavu during the last term of 2019. During that period, an abnormally high number of patients presented to local hospitals with symptoms of cough and fever, which were diagnosed as flu or atypical pneumonia for those who could have a chest radiography. Below are additional arguments that support the above hypothesis.

First, the COVID-19 epidemic has apparently been late to hit Africa, relative to the starting dates in Asia or Europe. This is not due to lack of contacts with China, known to be the origin of the pandemic. During the past two decades business exchanges between many regions of Africa, especially the eastern part of the continent, with Asia, and in particular with China, have been on the increase [26-28]. Even after the 
official declaration of the pandemic, many persons from Africa continued to travel to China, to secure the import of various goods. The apparent delay in the outbreak of COVID-19 cannot therefore be explained solely on the grounds that there is a low level of traveling between Africa and China $[14,28]$.

Secondly, despite the evidence of community spread of COVID-19 in DRC since more than seven months, the progression of the number of such cases has been relatively slower compared to Europe, the USA and Latin America. One reason for the small number of reported positive cases could arguably be due to the low level of testing. However, indirect evidence of the local propagation of the disease consists in the occurrence of an increased number of people consulting for cough, fever, dyspnoea and eventually for severe respiratory distress requiring respiratory assistance as it has been the case from the last week of May 2020 up to mid-July 2020 [20]. From mid-March when the first COVID-19 were reported in South-Kivu up to end-May, such an increased incidence of cases of acute respiratory distress was not observed locally, suggesting that there was either low levels of circulating Coronavirus before May or that there was epidemic spread of COVID-19 which remained asymptomatic or paucisymptomatic. The latter could probably be due to protective factors such as the generally young age of the population, other immunizations (Bacille Calmette-Guérin vaccination, measles, etc.), some environmental or genetic factors, etc.

In view of the above-mentioned observations, it is tempting to suggest that SARS-CoV-2 infection might have already been present in the region. If this were to be true, then it would raise the questions as to whether the pandemic really started in China in December 2019 and why the disease did not develop into an epidemic for so many months. Similar reports of COVID-19 cases before the declaration of the pandemic exist [29]. It is important to underline the limitations of using retrospective case reports as evidence of suspected COVID-19, especially in contexts such as ours with limited detailed information on travel/contact history and with limited conservation of blood or other samples for retrospective laboratory analysis.

\section{Conclusion}

This case report highlights a number of facts suggesting that COVID-19 may have already been present in parts of eastern DRC (and possibly other parts of East Africa) months before the official start of the pandemic. Prior infection with SARS-Cov2 or by other infections causing cross-immunity could explain the late and smaller extent of the COVID-19 pandemic in the region. We wish to carry out larger studies, including retrospective microbiological and serological investigations in local communities in order to test this hypothesis. There is also a need to develop more robust epidemiological surveillance systems in Africa, sensitive to the threat of pandemics as a consequence of increased international traffic and globalisation.

\section{Abbreviations}


Arterial blood gas

ARDS

Acute respiratory distress syndrome

COVID-19

The novel coronavirus disease 2019

CRP

C-reactive protein

CXR

Chest X-Rays

DRC

The Democratic Republic of the Congo

HIV

Human Immunodeficiency Virus

HPGRB

Hôpital Provincial Général de Référence de Bukavu

ICU

Intensive Care Unit

$\mathrm{SaO}_{2}$

Oxygen saturation

SARS-CoV-2

Severe acute respiratory syndrome coronavirus 2

\section{Declarations}

\section{Ethics approval and consent to participate}

All patients admitted in the "Hôpital Provincial Général de Référence de Bukavu" consent that their clinical and imaging data can be used for publication or education purpose. All procedures performed were in accordance with the ethical standards of the institutional ethical committee and with the 1964 Helsinki declaration and its later amendments.

All patients admitted consent that their clinical and imaging data can be used for publication or education purpose.

\section{Consent for publication}

Written informed consent for publication of the clinical details and/or clinical images was obtained from the patients or the next of kin (for the deceased patient).

\section{Availability of data and materials}

All data generated or analysed during this study are included in this manuscript. 


\section{Competing interests}

The authors declare that they have no competing interests.

\section{Funding}

There was no funding source for this study.

\section{Authors' contributions}

All authors contributed to the study conception and design. GQM, PM, SM, AB and BF contributed to the clinical patient care and management. Chest X-Rays were performed and interpreted by FN and GM. All authors contributed to the literature search. The first draft of the manuscript was written by MB and PM and all authors contributed to subsequent versions of the manuscript. All authors read and approved the final manuscript.

\section{Acknowledgements}

Not applicable.

\section{References}

1. Zhu N, Zhang D, Wang W, Li X, Yang B, Song J, et al. A Novel Coronavirus from Patients with Pneumonia in China, 2019. N Engl J Med. 2020;382:727-33. Available from: https://pubmed.ncbi.nlm.nih.gov/31978945.

2. World Health Organization (WHO). Coronavirus disease (COVID-19): Similarities and differences with influenza. Q\&A. 2020. [Accessed 2020 Nov 10]. Available from: https://www.who.int/news-room/q-adetail/coronavirus-disease-covid-19-similarities-and-differences-with-influenza.

3. Jung C-Y, Park H, Kim DW, Choi YJ, Kim SW, Chang TI. Clinical Characteristics of Asymptomatic Patients with COVID-19: A Nationwide Cohort Study in South Korea. Int J Infect Dis. 2020;99:266-8. Available from: https://pubmed.ncbi.nlm.nih.gov/32771632.

4. Huang C, Wang Y, Li X, Ren L, Zhao J, Hu Y, et al. Clinical features of patients infected with 2019 novel coronavirus in Wuhan, China. Lancet. 2020;395:497-506.

5. Wang D, Hu B, Hu C, Zhu F, Liu X, Zhang J, et al. Clinical Characteristics of 138 Hospitalized Patients With 2019 Novel Coronavirus-Infected Pneumonia in Wuhan, China. JAMA. 2020;323:1061-9. Available from: https://doi.org/10.1001/jama.2020.1585.

6. Chen N, Zhou M, Dong X, Qu J, Gong F, Han Y, et al. Epidemiological and clinical characteristics of 99 cases of 2019 novel coronavirus pneumonia in Wuhan, China: a descriptive study. Lancet. 2020;395:507-13.

7. Guan W, Ni Z, Hu Y, Liang W, Ou C, He J, et al. Clinical characteristics of coronavirus disease 2019 in China. N Engl J Med. 2020;382:1708-20. 
8. Chan JF-W, Yuan S, Kok K-H, To KK-W, Chu H, Yang J, et al. A familial cluster of pneumonia associated with the 2019 novel coronavirus indicating person-to-person transmission: a study of a family cluster. Lancet. 2020;395:514-23.

9. Mao L, Jin H, Wang M, Hu Y, Chen S, He Q, et al. Neurologic Manifestations of Hospitalized Patients With Coronavirus Disease 2019 in Wuhan, China. JAMA Neurol. 2020;77:683-90. Available from: https://doi.org/10.1001/jamaneurol.2020.1127.

10. Vaira LA, Salzano G, Deiana G, De Riu G. Anosmia and Ageusia: Common Findings in COVID-19 Patients. Laryngoscope. 2020;130:1787.

11. Wang J, Jiang M, Chen X, Montaner LJ. Cytokine storm and leukocyte changes in mild versus severe SARS-CoV-2 infection: Review of 3939 COVID-19 patients in China and emerging pathogenesis and therapy concepts. J Leukoc Biol. 2020;108:17-41. Available from:

https://pubmed.ncbi.nlm.nih.gov/32534467.

12. Johns Hopkins University. COVID-19 Dashboard by the Center for Systems Science and Engineering (CSSE) at Johns Hopkins University (JHU). Johns Hopkins Coronavirus Resour. Cent. 2020 [Accessed 2020 Nov 15]. Available from: https://coronavirus.jhu.edu/map.html.

13. Velavan TP, Meyer CG. The COVID-19 epidemic. Trop Med Int Heal. 2020;25:278-80.

14. Gilbert M, Pullano G, Pinotti F, Valdano E, Poletto C, Boëlle P, et al. Preparedness and vulnerability of African countries against importations of COVID-19: a modelling study. Lancet. 2020;395:871-7.

15. Moore M, Gelfeld B, Okunogbe A, Paul C. Identifying Future Disease Hot Spots: Infectious Disease Vulnerability Index. Rand Heal Q. 2017;6:5. Available from: https://pubmed.ncbi.nlm.nih.gov/28845357.

16. World Health Organization (WHO). Coronavirus disease (COVID-2019) Situation report 26. 2020 [Accessed 2020 Jun 16]. Available from: https://www.who.int/emergencies/diseases/novelcoronavirus-2019/situation-reports.

17. Kapata N, Ihekweazu C, Ntoumi F, Raji T, Chanda-Kapata P, Mwaba P, et al. Is Africa prepared for tackling the COVID-19 (SARS-CoV-2) epidemic. Lessons from past outbreaks, ongoing pan-African public health efforts, and implications for the future. Int J Infect Dis. 2020;93:233-6. Available from: https://pubmed.ncbi.nlm.nih.gov/32119980.

18. World Health Organization (WHO). COVID-19 Weekly epidemiological update - 10 November 2020. 2020 [Accessed 2020 Nov 14]. Available from: https://www.who.int/publications/m/item/weeklyepidemiological-update--10-november-2020.

19. Ministère de la Santé Publique - Comité Nationalde Coordinaationde préparationet de risposte à l'épidémie de la maladie à Coronavirus 2019. Bulletin épidémiologique COVID-19 N²76/2020 du 01/11/2020 + Hebdo S44/2020. Kinshasa; 2020. [Accessed 2020 Nov 14]. Available from: https://reliefweb.int/report/democratic-republic-congo/bulletin-epidemiologique-covid-19-n-2762020du-01112020.

20. Division Provinciale de la Santé Province du Sud-Kivu. Profil épidémiologique de la Province du SudKivu. Semaine 42. 21 octobre 2020. 2020. 
21. Mbow M, Lell B, Jochems SP, Cisse B, Mboup S, Dewals BG, et al. COVID-19 in Africa: Dampening the storm? Science. 2020;369:624-626. Available from:

http://science.sciencemag.org/content/369/6504/624.abstract.

22. Jacobi A, Chung M, Bernheim A, Eber C. Portable chest X-ray in coronavirus disease-19 (COVID-19): A pictorial review. Clin Imaging. Elsevier; 2020;64:35-42. Available from: https://doi.org/10.1016/j.clinimag.2020.04.001.

23. Chen T, Wu D, Chen H, Yan W, Yang D, Chen G, et al. Clinical characteristics of 113 deceased patients with coronavirus disease 2019: retrospective study. BMJ. 2020;368:m1091.

24. Guan W, Liang W, Zhao Y, Liang H, Chen Z, Li Y, et al. Comorbidity and its impact on 1590 patients with Covid-19 in China: A Nationwide Analysis. Eur Respir J. 2020;55(5):2000547.

25. Stewardson AJ, Grayson ML. Psittacosis. Infect Dis Clin North Am. 2010;24:7-25.

26. Zhou Y. Air traffic between China and Africa has jumped 630\% in the last decade. Quartz Africa. 2019 [Accessed 2020 Oct 24]. Available from: https://qz.com/africa/1675287/china-to-africa-flightsjumped-630-in-the-past-nine-years/.

27. Pirie G. China and aviation in Africa: context, thrust, novelty. African Geogr Rev. 2020;39:74-89. Available from: https://doi.org/10.1080/19376812.2019.1599294.

28. Lalaoui R, Bakour S, Raoult D, Verger P, Sokhna C, Devaux C, et al. What could explain the late emergence of COVID-19 in Africa? New Microbes New Infect. 2020;38:100760. Available from: http://www.sciencedirect.com/science/article/pii/S2052297520301128.

29. Deslandes A, Berti V, Tandjaoui-Lambotte Y, Alloui C, Carbonnelle E, Zahar JR, et al. SARS-CoV-2 was already spreading in France in late December 2019. Int J Antimicrob Agents. 2020;55:106006. Available from: http://www.sciencedirect.com/science/article/pii/S0924857920301643.

\section{Figures}



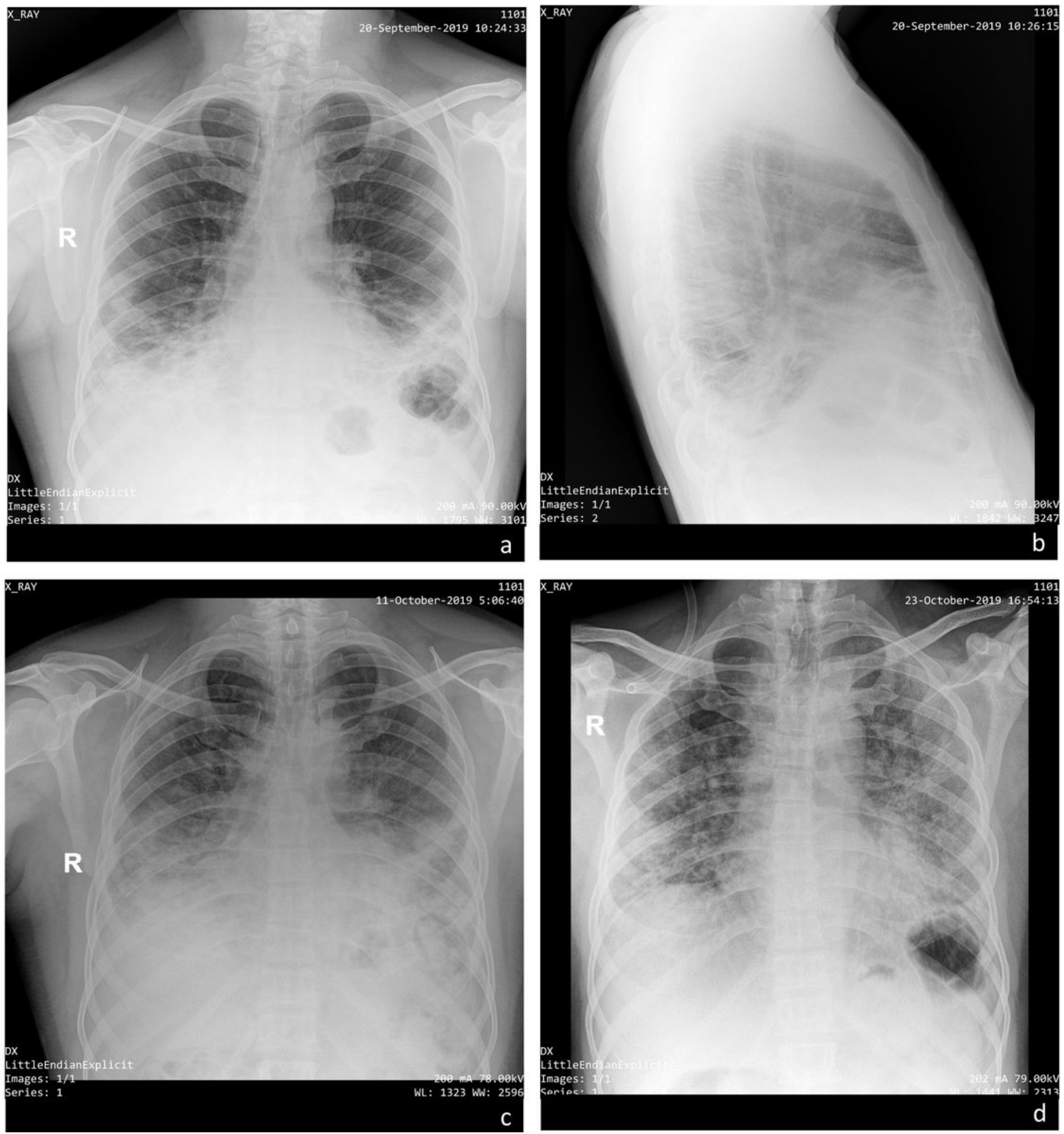

\section{Figure 1}

Serial Chest X-Rays of Patient 1. Multifocal then diffuse airspace disease in a 55-years-old man. Bibasal lung consolidation with assorted trabeculae, especially in the posterior regions, overhung by discreet hazy opacities $(a, b)$. Follow-up showing a cranially progress of the predominant inhomogeneous consolidation still coexisting with a clearly diffuse ground-glass (c). There is apparent replacement of alveolar densification by diffuse reticular interstitial marks with some few parahilar nodules (d) 

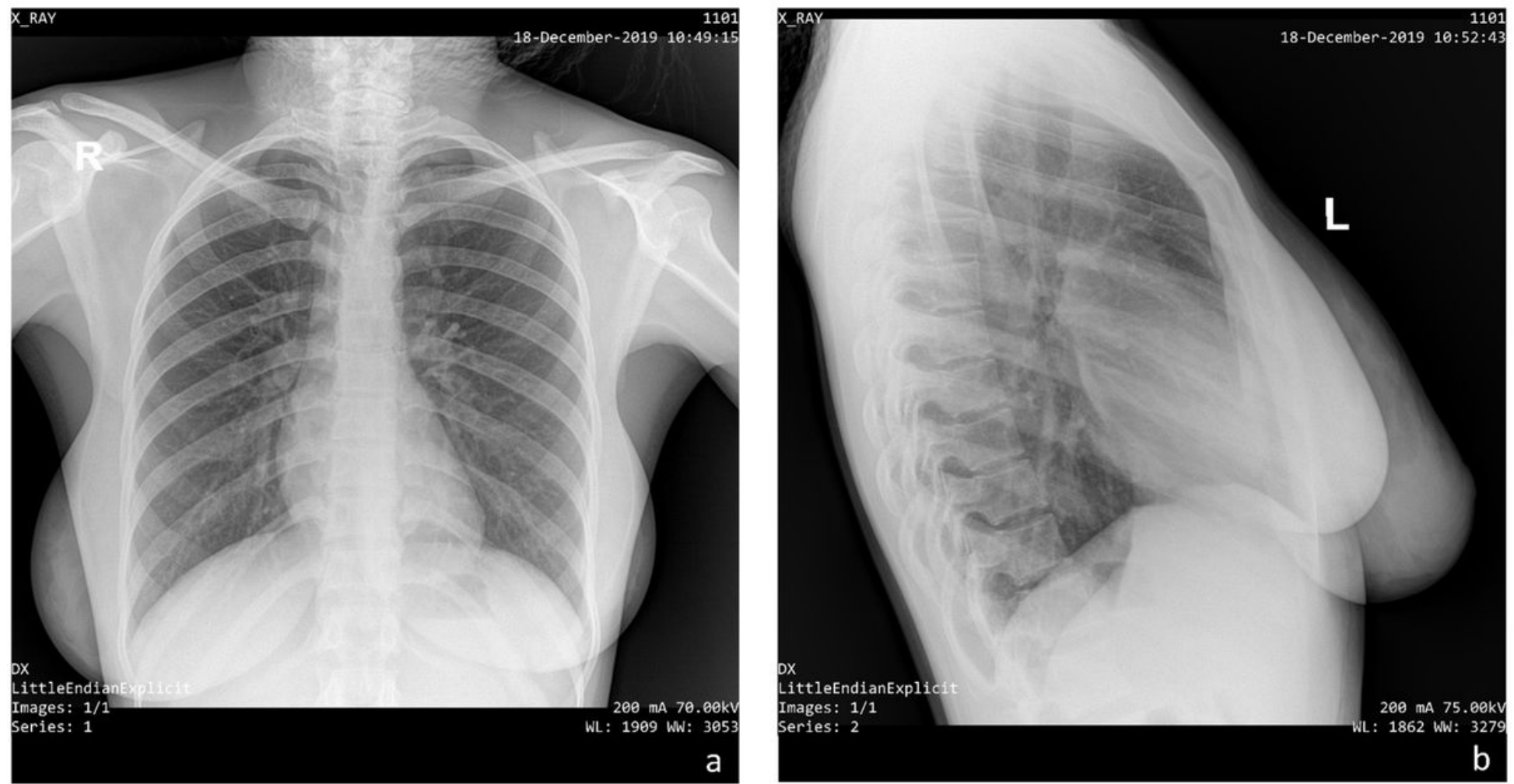

\section{Figure 2}

Chest X-Rays of Patient 2. Subnormal CXR of a 25-year-old female. Discrete peribronchovascular blur in the posterobasal regions. Normal aspect of the hila and the costophrenic angles.

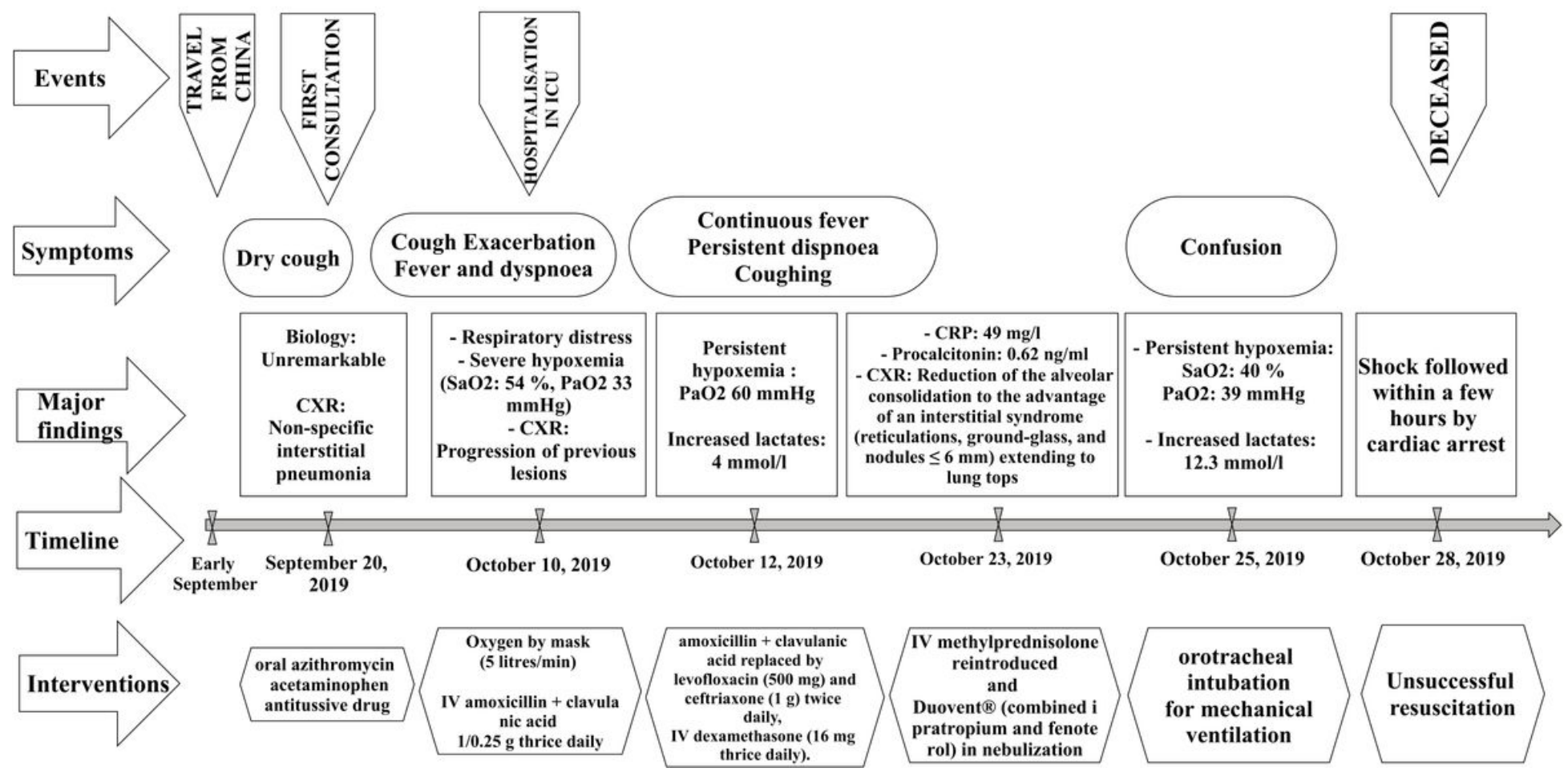

\section{Figure 3}




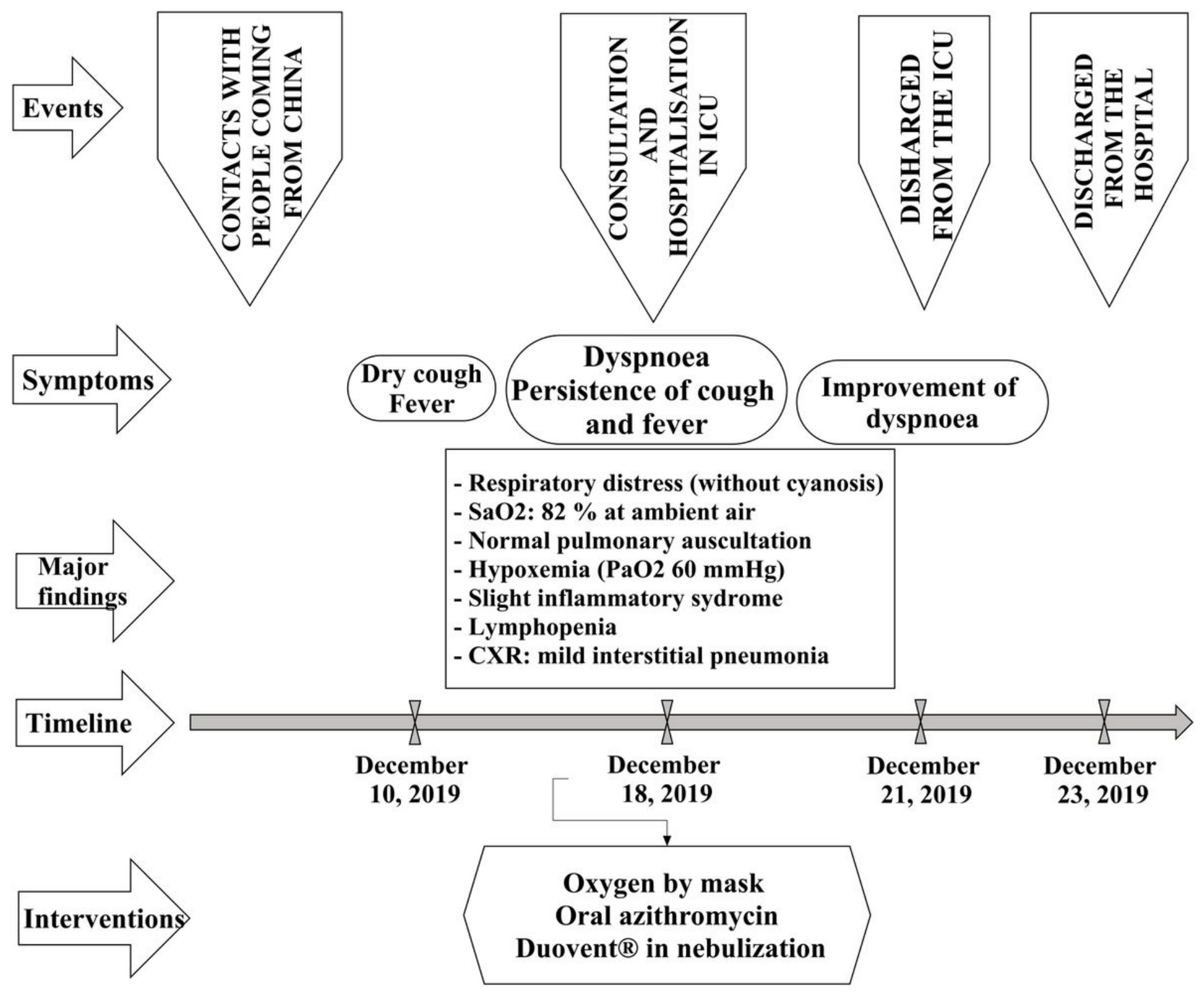

Figure 4

Summary of the 25 -year-old patient's information and care organized as a timeline 